\title{
Faint solar radio structures from decametric observations
}

\author{
C. Briand ${ }^{1}$, A. Zaslavsky ${ }^{2}$, M. Maksimovic ${ }^{1}$, P. Zarka ${ }^{1}$, A. Lecacheux ${ }^{1}$, H. O. Rucker ${ }^{3}$, A. A. Konovalenko ${ }^{4}$, \\ E. P. Abranin ${ }^{4}$, V. V. Dorovsky ${ }^{4}$, A. A. Stanislavsky ${ }^{4}$, and V. N. Melnik ${ }^{4}$ \\ 1 LESIA, Observatoire de Paris, CNRS, UPMC, Université Paris Diderot, 5 Place Jules Janssen, 92190 Meudon, France \\ e-mail: carine.briand@obspm.fr \\ 2 Laboratoire de Physique et Technologie des Plasmas, Ecole Polytechnique, 91128 Palaiseau Cedex, France \\ 3 Space Research Institute, Austrian Academy of Sciences, Schmiedlstrasse 68042 Graz, Austria \\ ${ }^{4}$ Institute of radio Astronomy, National Academy of Science of Ukraine, Ukraine
}

Received 25 March 2008 / Accepted 26 July 2008

\section{ABSTRACT}

\begin{abstract}
Aims. Decameter radio observations of the solar corona reveal the presence of numerous faint frequency drifting emissions, similar to "solar S bursts" which are reported in the literature. We present a statistical analysis of the characteristics of these emissions and propose a mechanism to excite the Langmuir waves thought to be at the origin of these emissions.

Methods. The observations were performed between 1998 and 2002 with the Digital Spectro Polarimeter (DSP) receivers operated at the UTR-2 and Nançay decameter radio telescopes in the frequency range 15-30 MHz. Our theoretical explanation is based on Vlasov-Ampère simulations.

Results. Based on the frequency drift rate, three populations of structures can be identified. The largest population presents an average negative frequency drift of $-0.9 \mathrm{MHz} \mathrm{s}^{-1}$ and a lifetime up to $11 \mathrm{~s}$ (median value of $2.72 \mathrm{~s}$ ). A second population shows a very small frequency drift of $-0.1 \mathrm{MHz} \mathrm{s}^{-1}$ and a short lifetime of about $1 \mathrm{~s}$. The third population presents an average positive frequency drift of $+0.95 \mathrm{MHz} \mathrm{s}^{-1}$ and a lifetime of up to $3 \mathrm{~s}$. Also, the frequency drift as a function of frequency is consistent with the former results, which present results in higher frequency range.

No specific relationship was found between the occurrence of these emissions and the solar cycle or presence of flares.

Assuming that these emissions are produced by "electron clouds" propagating the solar corona, we deduce electron velocities of about 3-5 times the electron thermal velocity. As previously shown, a localized, time-dependent modulation of the electron distribution function (heating) leads to low velocity electron clouds (consistent with observations), which, in turn, can generate Langmuir waves and electromagnetic signals by nonlinear processes.
\end{abstract}

Key words. Sun: radio radiation - Sun: Corona - Sun: particle emission - methods: numerical

\section{Introduction}

Decameter radio observations of the solar corona reveal the presence of numerous types of emission among which the well studied type I, II, III, and IV bursts and storms. These intense emissions spread over a wide range of frequencies and usually exhibit fine structures (see Goldman 1983, for a review of type III emission; and Dulk 1985, for a general review of solar radio emission). Chains of stria of type III like bursts, named type IIIb (Ellis \& McCulloch 1967; de La Noe \& Boischot 1972) are frequently observed and appear to be precursors of type III emission. Emissions of short duration and limited frequency extent are also detected as isolated drift pairs (Roberts 1958; Warwick \& Dulk 1969; de La Noe \& Moller Pedersen 1971; Melnik et al. 2005), consisting of fundamental and harmonic radio emission, usually related to the presence of type III bursts.

High spectral and temporal resolution observations have revealed that the decameter range is also rich in faint intensity types of emission (Barrow et al. 1994). These structures can provide some indications of the physical processes taking place on small spatial scales. Single drift bursts of the same characteristics (occurrence, frequency drift) as the drift pairs were reported (de La Noe \& Moller Pedersen 1971). Their emission is interpreted as the fundamental of the drift pairs. However, the isolated emission we are interested in is not related to the presence of drift pairs. The emission we study has characteristics similar to those of the "Fast Drift Bursts" (Ellis 1969), which were later referred as solar "S bursts" by McConnell (1980). A detailed analysis of these types of emission was presented by McConnell (1982) in the frequency range 40-80 MHz. Since then, this emission deserved little attention due to the difficulties in its observation.

The type II and III emission are explained in terms of electron beams propagating the interplanetary medium (Ginzburg \& Zhelezniakov 1958). As they propagate, the electron beams excite electrostatic Langmuir waves, which couple each other to generate to electromagnetic waves at the fundamental of the local plasma frequency ${ }^{1}$. The drift in frequency with time is a consequence of the decrease in density (and the plasma frequency) with distance from the Sun. The electron acceleration mechanism differs however for type II and type III emissions. For type II emission, electrons are accelerated by shock propagating at thousands of km/s ahead of a Coronal Mass Ejection (CME). For type III emission, acceleration of electron beams is thought to be related to magnetic reconnection and energy release during flares. Both types of emission involve high velocity electron

\footnotetext{
1 If this basic mechanism remains valid, a process should be proposed to limit the wave growth and retain the electron beams over long distances. This question is however outside the scope of this paper.
} 
Table 1. Observation characteristics and instrumental configuration. First column: observing date; second column: radiotelescope used ("N") for Nançay and "K" for Kharkov) with the time resolution in parenthesis (in seconds); third column: start time of the observing run (in Universal Time); fourth colum: end time of the run (in UT); fifth column: frequency range of the observations; sixth column: number of drifting structures identified.

\begin{tabular}{|c|c|c|c|c|c|}
\hline Date & $\begin{array}{l}\text { Instr. } \\
\text { (s) }\end{array}$ & $\begin{array}{c}\text { Start time } \\
\text { UT }\end{array}$ & $\begin{array}{c}\text { End time } \\
\text { UT }\end{array}$ & $\begin{array}{c}\text { Frequency } \\
\mathrm{MHz}\end{array}$ & $\begin{array}{c}\text { Structures } \\
\#\end{array}$ \\
\hline May 2, 1998 & $\mathrm{~N}(0.05)$ & $13: 28: 10$ & $13: 44: 18$ & $33-46$ & 0 \\
\hline June 6, 1999 & $\mathrm{~K}(0.05)$ & $08: 33: 50$ & $08: 59: 54$ & $15-28$ & 0 \\
\hline June 26, 1999 & $\begin{array}{l}\mathrm{K}(0.20) \\
\mathrm{K}(0.10)\end{array}$ & $\begin{array}{l}10: 14: 34 \\
11: 27: 20\end{array}$ & $\begin{array}{l}10: 22: 58 \\
12: 00: 40\end{array}$ & $\begin{array}{l}11-24 \\
11-24\end{array}$ & 258 \\
\hline June 27 , & $\mathrm{K}(0.10)$ & $08: 55: 30$ & $11: 43: 20$ & $11-24$ & 415 \\
\hline June 28, 1999 & $\mathrm{~K}(0.10)$ & 09:04:20 & 10:08:20 & $11-24$ & 9 \\
\hline July 1, 1999 & $\mathrm{~K}(0.02)$ & $09: 16: 23$ & $09: 58: 40$ & $11-24$ & 45 \\
\hline July 2,1 & $\mathrm{~K}(0.02)$ & 09:51:52 & $10: 08: 45$ & $11-24$ & 53 \\
\hline July 3, 1999 & $\mathrm{~K}(0.02)$ & $10: 05: 52$ & $10: 12: 59$ & $11-24$ & 16 \\
\hline Aug. 3, 1999 & $\mathrm{~N}(0.10)$ & 09:49:58 & $12: 29: 14$ & $18-30$ & $1(?)$ \\
\hline Feb. 27, 2000 & $\mathrm{~N}(0.10)$ & $09: 17: 02$ & $11: 49: 40$ & $33-46$ & $\overline{0}$ \\
\hline July 12,2002 & $\bar{K}(0.05)$ & $07: 04: 15$ & $10: 41: 09$ & $18-29$ & 51 \\
\hline July 13,2002 & $\mathrm{~K}(0.05)$ & $06: 23: 37$ & $11: 16: 52$ & $18-29$ & 808 \\
\hline
\end{tabular}

beams $(0.2 c$ to $0.6 c)$ and their occurrence is strongly correlated with solar activity (and therefore the solar cycle).

This paper presents a statistical analysis of faint, isolated, drifting solar emission in the range $15-30 \mathrm{MHz}$, extending the work of McConnell (1982) to the low frequencies of the decameter domain. Our set of observations covers an extended period of time around the last solar maximum (1998-2002). In Sect. 2, we present the instruments and data selection procedure used for the analysis. The results of the statistical analysis are described in Sect. 3. The characteristics of the emission suggest that electron beams propagating outwards (and inwards) through the solar corona could be the origin of the emission. The physical properties of these beams are determined in Sect. 4. Finally, Sect. 5 discusses the results and proposes a physical mechanism to explain the generation of Langmuir waves from low velocity electron beams.

\section{Observations and selection procedure}

\subsection{Observations}

The observations were performed at the UTR-2 Kharkov decameter array in Ukraine (Braude et al. 1978) and the Nançay decameter array in France (Boischot et al. 1980). The two radio telescopes were equipped with a Digital Spectro Polarimeter (DSP, Lecacheux et al. 1998) allow us to analyze the spectral range 15-33 MHz within 1024 channels. The main characteristic of the DSP is a temporal resolution of 50 milliseconds per spectrum. Combined with a dynamic range of $70 \mathrm{~dB}$, the DSP is well adapted to observe the very faint and short structures of the solar corona. Polarization measurements are available from the Nançay array but this information is not used in the present study (because no faint structures was detected).

Several long series of observations are analyzed in the present work. Table 1 summarizes the characteristics of each observing run. They are distributed about the last solar maximum (between May 1998 and July 2002).

\subsection{Structures selection}

The analysis is based on time-frequency images (dynamic spectra). Each image covers a range of $50 \mathrm{~s}$ in time and $11 \mathrm{MHz}$ in frequency. Figure 1 shows an example of these images.

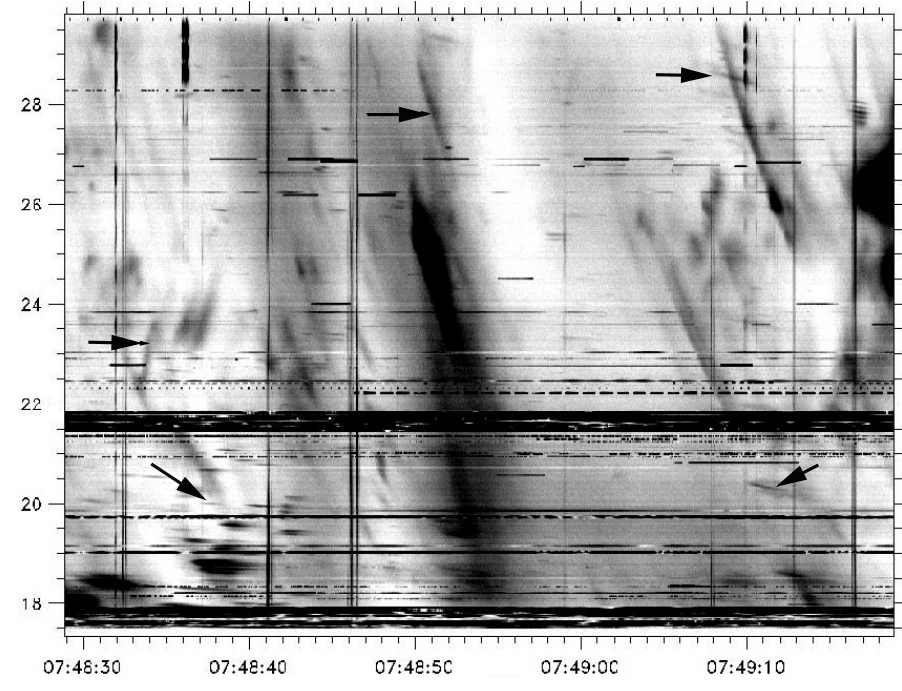

Fig. 1. Example of dynamic spectra from UTR-2 from the time series of July, 13 2002. Examples of the drifting structures studied here are indicated by arrows.

Due to the variety of their shapes (length, time profile, slope) and their low intensity level, each structure had to be identified by manual selection of several points along the crest line. The following selection criteria were used:

$$
\begin{aligned}
& \text { - continuity in time and frequency; } \\
& \text { - start and end points clearly identified (the drifting structures } \\
& \text { starting or ending inside a strong emission were rejected); } \\
& \text { - low intensity level. }
\end{aligned}
$$

This selection procedure introduces some biases. First, the starting and ending point locations depend strongly on the contrast in images. If the intensity is too low, these points may be undistinguishable from the background. Also, some structures start or end too close to parasitic emissions. These two errors lead to an underestimation of the actual time duration of the emission, which is difficult to quantify. Another error comes from the manual selection along the structure estimated to be $0.5 \mathrm{~s}$ in time and $0.1 \mathrm{MHz}$ in frequency. To reduce the impact of these uncertainties on the frequency drift computation, each structure is fitted by a straight line. The result of the fitting determines the frequency drift.

Another bias in the selection procedure is the difficulty in distinguishing between one long structure with intensity variations and two distinct structures. This implies that there is an underestimation in the length of some structures. The number of such cases is however limited and in cases of doubt the emission was rejected.

Finally, emission with a positive drift was usually more difficult to identify due to its low intensity, leading to an underestimation of its population.

In spite of these limitations, some significant characteristics of the emission can be obtained (see below). When present, drift pairs were included in the statistics. As we see, their characteristics are similar to the faint structures that we are interested in.

The frequency associated with each structure is the starting point frequency, which provides information about the altitude of emission in the solar corona. The length in time and frequency is determined from the selected ending points.

Table 1 indicates the total number of structures identified in each observing run. In the following, the characteristics (time 


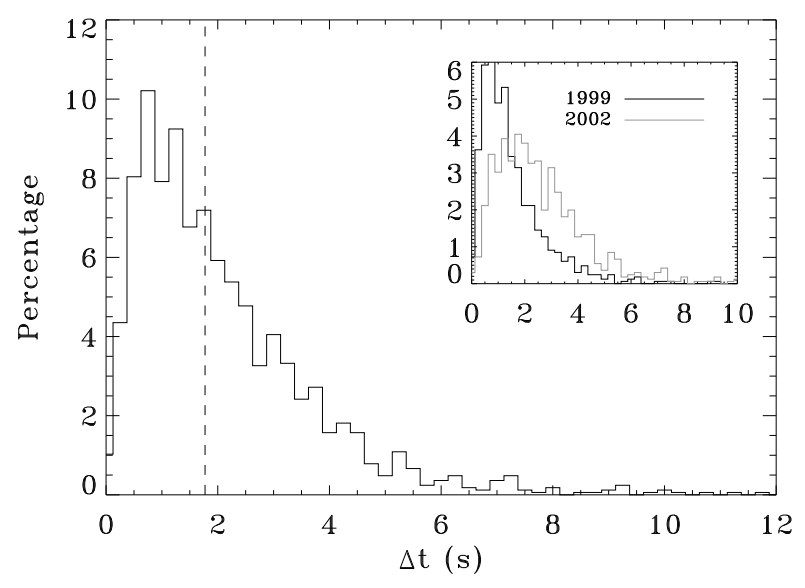

Fig. 2. Histogram of the time duration for the entire set of drifting structures. The vertical dashed line indicates the median value. The inset shows the same histogram, distinguishing between the observations of 1999 and 2002 (normalized to the total number of emissions).

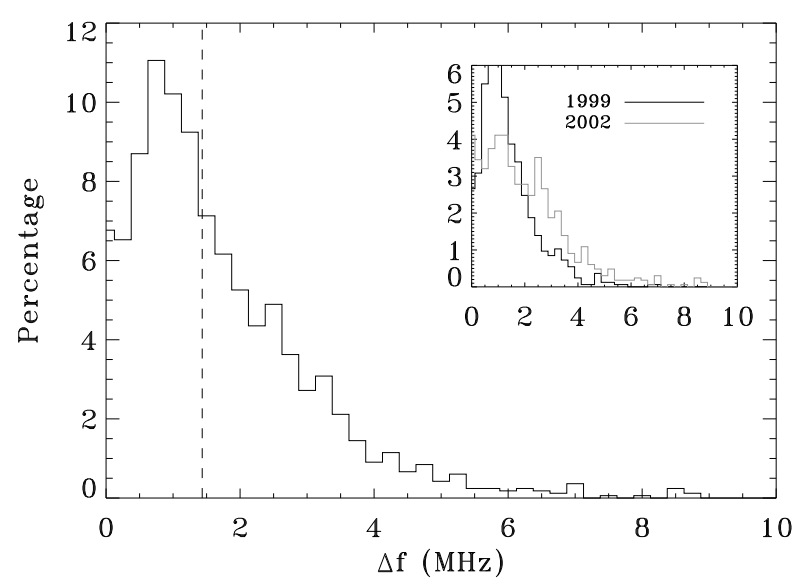

Fig. 3. Histogram of the frequency extent of drifting structures. The vertical dashed line indicates the median value. The inset shows the same histogram, but with the observations of 1999 and 2002 being displayed separately.

duration, frequency length, frequency drift) are deduced from a statistical analysis of the 1655 identified structures.

\section{Characteristics of the emission}

The histogram of the time duration of each emission is presented in Fig. 2. The median value is $1.75 \mathrm{~s}$.

The histogram of the frequency extent is shown in Fig. 3. The median value is $1.4 \mathrm{MHz}$.

The emission is shorter in time and more narrow-banded than either type II to IV emission, but comparable to both drift pairs and $\mathrm{S}$ bursts, which justifies the inclusion of these last two types of emission in our study.

The histogram of frequency drifts (Fig. 4) reveals three distinct populations: the largest population (Population 1: 63\% of the sample) has a median value of $-0.9 \mathrm{MHz} \mathrm{s}^{-1}$; a second one (Population 2: $12 \%$ of the sample) has a median value of $-0.10 \mathrm{MHz} \mathrm{s}^{-1}$; and a third (Population 3: 23\% of the sample) has a median value of $+1.1 \mathrm{MHz} \mathrm{s}^{-1}$. The remaining $2 \%$ consists

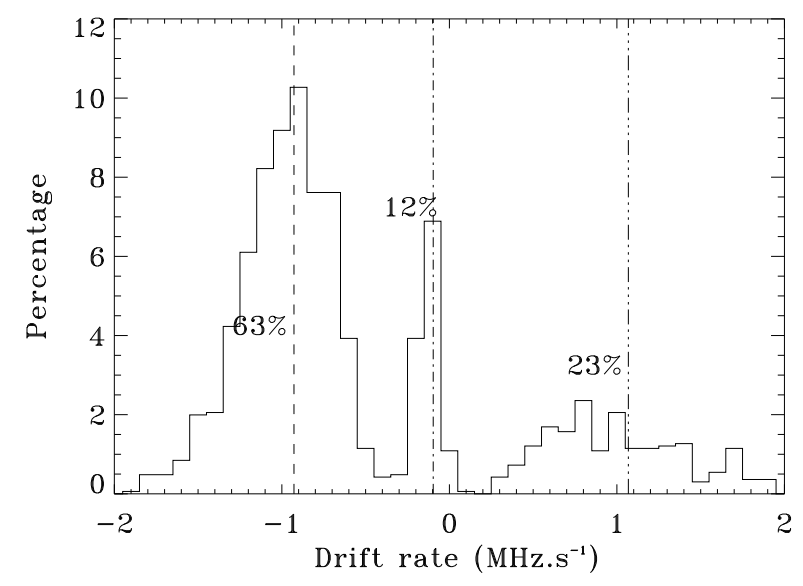

Fig. 4. Histogram of the frequency drift rate expressed as a percentage of the total number of structures. The median value of each population is indicated by the vertical lines. For sake of clarity, only structures between $\pm 2 \mathrm{MHz} \mathrm{s}^{-1}$ are displayed.

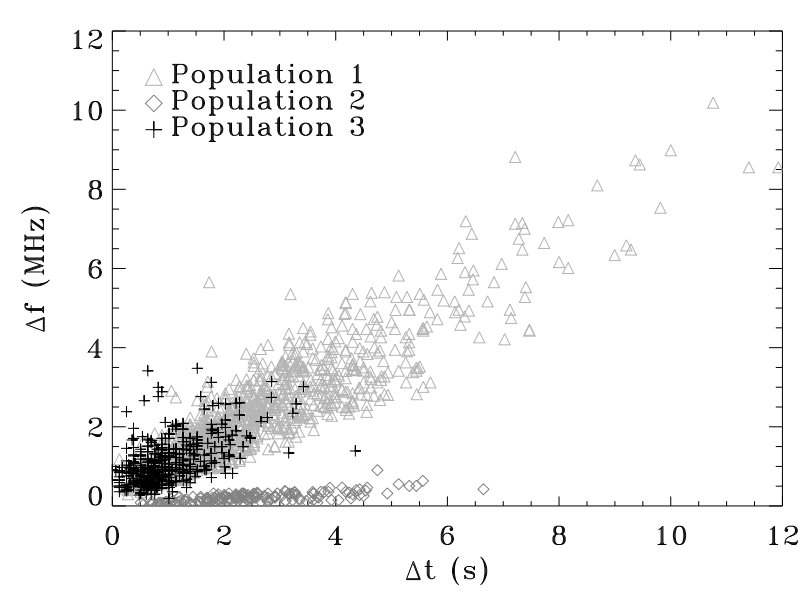

Fig. 5. Frequency extent as a function of the time duration of the structures. The three populations discussed in the text (see also Fig. 4) are distinguished by different symbols.

of structures with velocities scattered above $+2 \mathrm{MHz} \mathrm{s}^{-1}$. The presence of structures with reverse drift (towards higher frequencies) was also mentioned (for example by Ellis 1969; McConnell 1982) but not studied in detail, probably due to the lack of high sensitivity instruments. Also, the sporadic nature of this emission make it difficult to detect. The relationship between the frequency extent and the duration is shown in Fig. 5. A first group (crosses and triangles on the graph) presents a linear variation with a slope of $0.76 \mathrm{MHz} \mathrm{s}^{-1}$, while a second group (diamond on the graph) shows a slope of $0.03 \mathrm{MHz} \mathrm{s}^{-1}$. This latter represents only $9 \%$ of the entire sample.

The three populations discussed previously (and shown in Fig. 4) are separated well in Fig. 5: population 2 (small negative drift) corresponds to the almost horizontal structures, while populations 1 and 3 have the same steeper slope. Population 3 consists of structures with the shortest extent (both in time and frequency).

Frequency drifts are plotted as a function of frequency in Fig. 6 (left). We first note that the emission appears at all studied frequencies. Drift distributions within populations 1 and 

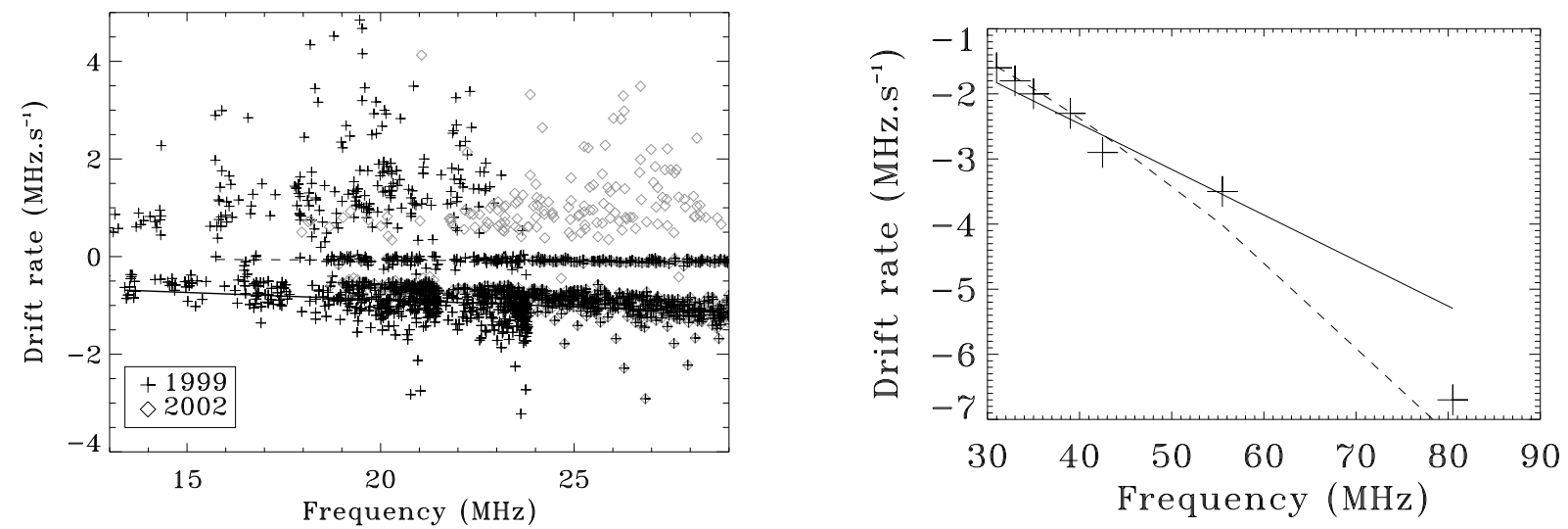

Fig. 6. Drift rate as a function of the frequency. Left: from our observation. Linear fits are indicated for populations 1 and 2. Right: from Table II of McConnell (1982). The straight line is the extent of the fit deduced from our data, the dashed line correspond to the fit of McConnell paper.

Table 2. Summary of the solar activity during the observing runs (in bold, flare starting after the end of our run). First column: observing date; second column: number of structures selected from the full observing run; third column: time (UT) of the maximum of H $\alpha$ Flares with in parenthesis the duration (in minutes); fourth column: time of maximum (in UT) of X-ray Flares followed by the class and duration (in minutes); fifth: time of occurrence of type III radio emission. "NA" means "Not available" information while dashes mean "no activity" in the corresponding emission. Sources for flares: Solar Geophysical Data; source for the radio emission: our observations.

\begin{tabular}{ccccc}
\hline \hline Date & $\begin{array}{c}\text { Structures } \\
\#\end{array}$ & $\begin{array}{c}\text { H } \alpha \text { flare } \\
\text { Time (duration) }\end{array}$ & $\begin{array}{c}\text { X-rays flares } \\
\text { Time (Class; duration) }\end{array}$ & $\begin{array}{c}\text { type III } \\
\text { Time }\end{array}$ \\
\hline May 2, 1998 & 0 & $13: 28(20)$ & $13: 31(\mathrm{X} 1.1 ; 20)$ & $13: 38$ \\
\hline June 6, 1999 & 0 & $08: 55(3)$ & - & $08: 51$ \\
June 26, 1999 & 258 & - & $11: 39(\mathrm{C} 3.1 ; \mathrm{NA})$ & $11: 49$ \\
June 27, 1999 & 415 & $09: 07(60)$ & - & $11: 34$ \\
June 28, 1999 & 9 & $09: 21(8), 09: 21(7)$ & - & $09: 06,09: 08,09: 16$ \\
& & - & - & $09: 19,09: 57,10: 07$ \\
July 1, 1999 & 45 & $10: 15(20)$ & - & $09: 23$ \\
July 2, 1999 & 53 & - & $11: 40(\mathrm{C} 4.6 ; 14), 12: 25(\mathrm{C} 3.1 ; 8)$ & - \\
July 3, 1999 & 16 & $09: 47(8), 10: 32(10)$ & - & $09: 53,10: 09,10: 30,10: 42$ \\
August 3, 1999 & $1(?)$ & - & - & $11: 20,12: 16,12: 24$ \\
\hline Feb. 27, 2000 & 0 & $-08: 51(14)$ & $06: 59(\mathrm{C} 1.7 ; 28), 08: 56(\mathrm{C} 1.6 ; 12), 10: 35(\mathrm{~B} 8.4 ; 6)$ & $09: 59$ \\
\hline July 12, 2002 & 51 & $07: 35(19), 07: 53(4)$ & $07: 53(\mathrm{C} 1.6 ; 5), 08: 15(\mathrm{C} 2.2 ; 6)$ & $08: 00,10: 17$ \\
July 13, 2002 & 808 & $08: 15(9)$ & & $06: 29,07: 31$ \\
& & &
\end{tabular}

2 themselves drift at $-0.07 \mathrm{MHz} \mathrm{s}^{-1} / \mathrm{MHz}$ for population 1 and $-5 \times 10^{-3} \mathrm{MHz} \mathrm{s}^{-1} / \mathrm{MHz}$ for population 2 . The positive values do not exhibit any distinctive trend. The measurements presented by de La Noe \& Moller Pedersen (1971, see their Fig. 2) agree with our own set of data, at least in the range of frequencies studied here. It seems, however, that their slope at negative values is larger (but their set of observation is limited). Figure 6 (right) shows the drift rate as a function of time from McConnell (1982), who explored the complementary domain 30-82 MHz. The fit of the current study is extrapolated towards the higher frequencies and superimposed on the fit proposed by McConnel $\left(\mathrm{d} f / \mathrm{d} t=-a f^{b}\right.$ with $a=6.5 \times 10^{-3}$ and $\left.b=1.60\right)$. The results of the two studies are consistent except for the last point at $80 \mathrm{MHz}$ where a strong decrease in the drift rate appears that is not evident in our set of data.

\subsection{Link with solar cycle and solar activity}

Drift pairs appear to be precursors of type III bursts, which means that their occurrence is related to solar activity. It is therefore interesting to test if the isolated, faint emission, which shares common characteristics with drift pairs, is dependent on any kind of activity or solar cycle.

In Figs. 2 and 3, the duration and frequency extent of the emission is distinguished for the two years 1999 and 2002. A slight tendency towards longer duration is present in 2002. However, no difference in drift rate is apparent in Fig. 6. The coverage of the last solar maximum is too incomplete to be conclusive about the dependence on solar cycle. More investigation is required to draw any conclusion.

The relationship between emission occurrence and solar activity is analyzed in Table 2 which presents a summary of the solar activity recorded during each observing run. Flares in $\mathrm{H} \alpha$, $\mathrm{X}$-rays, and type III allow us to appreciate the activity in the chromosphere, the corona and the propagation of electron beams in the interplanetary medium. Of course, decameter radio emission are characteristics of the high altitude corona (see Sect. 4) but activity in the lower atmosphere of the Sun can generate instabilities that propagate into the higher solar layers and produce radio emission.

The strong X-ray flare of May 2, 1998 or the more moderate ones of August 3, 1999 apparently did not produce any faint 
structure. However, only the Nançay instrument was observing at that time and it did not detect any structure, which may simply be due to a too high sensitivity threshold. On July 2 and 3, 1999, 69 structures were detected while no specific solar activity was present during the observing run. On July 12, 2002, 51 structures were detected while the activity was quite significant (3 X-ray flares, $1 \mathrm{H} \alpha$ flare and 2 type III), while on the next day, 808 structures were observed while the solar activity was limited to the beginning of the observation run. It therefore appears that the faint, isolated, drifting emission is not related to any specific solar activity. However, our set of observations is limited. In particular, the data set for 2002 consists of only two days of observations.

\section{Interpretation}

Due to their similarity with other decameter radio bursts (type III, drift pairs), we assume that this type of radio emission is the signature of electron beams propagating in the solar corona. Assuming that the observed emission is at the fundamental of the plasma frequency and given an electron density model, the beam velocity can be deduced from the observations.

The plasma frequency $f_{\mathrm{p}}$ is given by:

$f_{\mathrm{p}}=A \sqrt{n(r)}$

with $A \simeq 9$ for frequencies in $\mathrm{kHz}$ and density (as a function of the distance $r$ to the sun) $n(r)$ in $\mathrm{cm}^{-3}$. The frequency drift can thus be written:

$\frac{\mathrm{d} f}{\mathrm{~d} t}=\frac{\mathrm{d} f}{\mathrm{~d} n} \frac{\mathrm{d} n}{\mathrm{~d} r} \frac{\mathrm{d} r}{\mathrm{~d} t}$

which leads to the following expression, assuming a constant velocity $V$ of the beam:

$\frac{\mathrm{d} f}{\mathrm{~d} t}=\frac{A \cdot V}{2 \sqrt{n(r)}} \frac{\mathrm{d} n}{\mathrm{~d} r}$

The velocity $V$ can be derived from an a priori known density model. Since the corona is highly structured, no unique density model can describe it. Among the many existing models that can be found in the literature, we have chosen to use three: the model of Leblanc et al. (1998) of the solar corona and interplanetary medium (deduced from coronographic observations and type III radio bursts), the model of Newkirk (1961) of solar quiet corona, and the model of Doyle et al. (1999) of a solar coronal hole. Alvarez \& Haddock (1973) also derived electron densities from type III bursts, but their model is applicable mainly to large distances from the Sun. Mann et al. (1999) also proposed another density model which agrees to within $15 \%$ with the model of Newkirk in the range of altitude we are interested in. The density stratification corresponding to each model is shown in Fig. 7. The altitude range in the solar atmosphere covered by the frequency range of our observations (indicated by the horizontal grey lines) spans between 1.3 and 2.2 solar radii from solar mass center.

The three selected models provide similar results. Figure 8 displays the beam velocity as a function of the distance to the Sun, as obtained from the model of Leblanc et al. (1998).

We first check that the assumption allowing us to deduce Eq. (3) (constant beam velocity) is reasonable. The three models provide values below $0.1 c\left(0.1 c \simeq 10 \times v_{\text {te }}, v_{\text {te }}\right.$ being the thermal velocity of the electrons at this range of altitudes). The median velocity deduced from the models is given in Table 3 for each

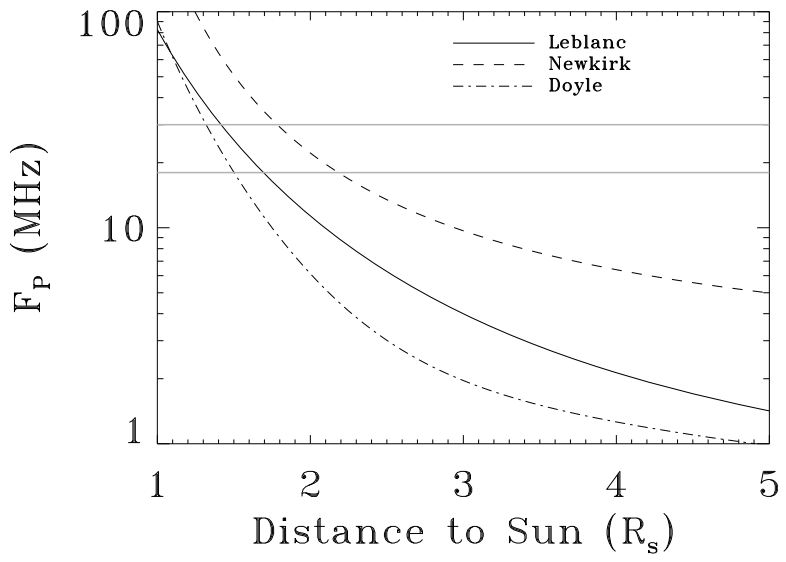

Fig. 7. Density stratification expressed in term of plasma frequency for the three models considered. The two grey horizontal lines delimitate the range of frequencies covered by our observations.

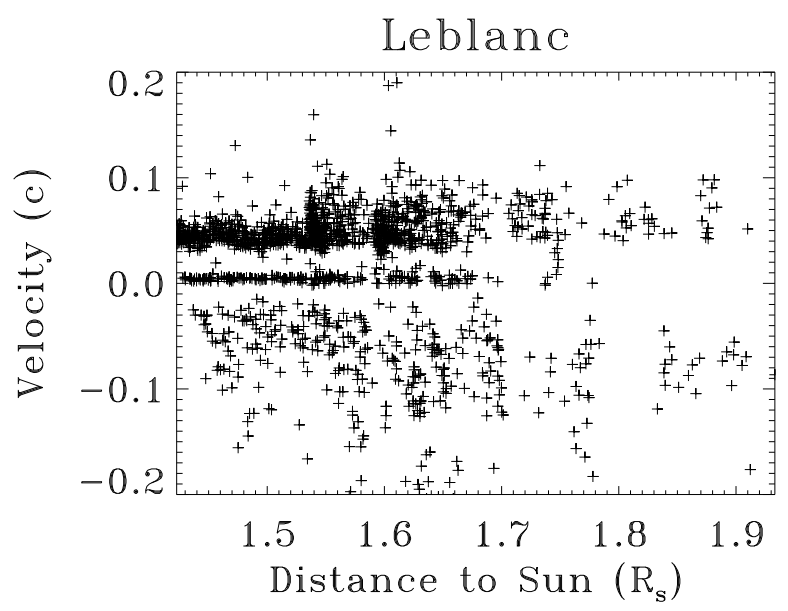

Fig. 8. Velocity of the observed structures (expressed in term of velocity of light) as a function of the distance to the Sun from the density model of Leblanc et al. (1998).

Table 3. Median value of the beam velocity deduced from each density model. Negative velocities are in the direction towards the Sun.

\begin{tabular}{cccc}
\hline \hline Model & Newkirk & Leblanc & Doyle \\
\hline$V>5 \times 10^{-3}(\mathrm{c})$ & +0.06 & +0.04 & +0.03 \\
$V \approx 0$ (c) & $+6 \times 10^{-3}$ & $+4 \times 10^{-3}$ & $+3 \times 10^{-3}$ \\
$V<-5 \times 10^{-3}$ (c) & -0.07 & -0.05 & -0.09 \\
\hline
\end{tabular}

population. Finally, we recall that in an atmosphere of $10^{6} \mathrm{~K}$, the thermal velocity is about $0.01 \mathrm{c}$. This means that the beams that we are considering travel at a few times the thermal velocity.

\section{Discussion}

The characteristics of the decameter emission that we have studied show that they are produced at all ranges of altitudes in the solar corona corresponding to the range 18-30 MHz (from about 1 to 2 solar radii). Most emission presents a drift rate suggesting a propagation of the source outwards from the Sun. The extent in frequency and time of these structures can be quite large (up to $10 \mathrm{MHz}$ and $11 \mathrm{~s}$, respectively). Emission with positive velocity drift also suggests a propagation of the source towards 
the Sun. However, in contrast to the previous group the extent in time and frequency is limited, which could indicate absorption in the denser layers of the solar atmosphere, close to the emission point.

In all cases, the frequency drift rate is far lower than those associated with type III radio bursts. If we admit that the same mechanism holds for the direct and reverse drift emissions, the electron beams generating the Langmuir waves at the origin of the radio emission must have velocities far smaller than the beams generating the type III emission. From standard coronal density models, we deduce velocities of a few times the electron thermal velocity. The electron beams at the origin of the type III radiobursts are thought to be generated by reconnection processes. This mechanism produces velocities at least 10 times higher than the velocity deduced from the present observations. We propose instead a moderate, localized, time-dependent heating to be at the origin of the Langmuir waves. As discussed in Briand et al. (2007), this mechanism yields electron clouds that can propagate, following a ballistic trajectory, at velocities of a few electron thermal velocity and generate Langmuir waves. If this interpretation is correct, this faint emission would be the signature of local plasma heating events in the corona. Population 2 emission processes must be different, since bump-in-tail instabilities (the basic instability that produces the Langmuir waves) are inefficient for beams of too low velocity. Processes similar to those implicated in the solar type IIIb are more likely to be at the origin of the isolated almost non drifting structures.

Since the mechanism that we propose does not require strong heating, the emission should correlated to neither solar activity nor solar cycle as actually observed. The present study appears to support this idea. However, more observations are required to be conclusive on this point.

Acknowledgements. This work was partly realized within the framework of the INTAS project N97-1964.

\section{References}

Alvarez, H., \& Haddock, F. T. 1973, Sol. Phys., 29, 197

Barrow, C. H., Zarka, P., \& Aubier, M. G. 1994, A\&A, 286, 597

Boischot, A., Rosolen, C., Aubier, M. G., et al. 1980, Icarus, 43, 399

Braude, S. I., Men, A. V., \& Sodin, L. G. 1978, Antenny, 26, 3

Briand, C., Mangeney, A., \& Califano, F. 2007, Phys. Lett. A, 368, 82

de La Noe, J., \& Moller Pedersen, B. 1971, A\&A, 12, 371

de La Noe, J., \& Boischot, A. 1972, A\&A, 20, 55

Doyle, J. G., Teriaca, L., \& Banerjee, D. 1999, A\&A, 349, 956

Dulk, G. A. 1985, ARA\&A, 23, 169

Ellis, G. R. A. 1969, Aust. J. Phys., 22, 177

Ellis, G. R. A., \& McCulloch, P. M. 1967, Aust. J. Phys., 20, 583

Ginzburg, V. L., \& Zhelezniakov, V. V. 1958, SvA, 2, 653

Goldman, M. V. 1983, Sol. Phys., 89, 403

Leblanc, Y., Dulk, G. A., \& Bougeret, J.-L. 1998, Sol. Phys., 183, 165

Lecacheux, A., Rosolen, C., Clerc, V., et al. 1998, in Advanced Technology MMW, Radio, and Terahertz Telescopes, ed. T. G. Phillips, Proc. SPIE, 3357, 533

Mann, G., Jansen, F., MacDowall, R. J., Kaiser, M. L., \& Stone, R. G. 1999, A\&A, 348, 614

McConnell, D. 1980, Proc. Astron. Soc. Aust., 4, 64

McConnell, D. 1982, Sol. Phys., 78, 253

Melnik, V. N., Konovalenko, A. A., Dorovskyy, V. V., et al. 2005, Sol. Phys., 231,143

Newkirk, G. J. 1961, ApJ, 133, 983

Roberts, J. A. 1958, Aust. J. Phys., 11, 215

Warwick, J. W., \& Dulk, G. A. 1969, ApJ, 158, L123 